\title{
MLL5 improves ATRA driven differentiation and promotes xenotransplant engraftment in acute promyelocytic leukemia model
}

\author{
Diego A. Pereira-Martins 1,2,3,4, Isabel Weinhäuser 1,2,3, Juan Luiz Coelho-Silva $\mathbb{1}^{2,4,5}$, Pedro L. França-Neto ${ }^{4}$, \\ Luciana Y. Almeida ${ }^{2}$, Thiago M. Bianco $\mathbb{B}^{2,5}$, Cleide L. Silva ${ }^{2}$, Rafael F. França $\mathbb{B}^{6}$, Fabiola Traina $\mathbb{C}^{2,5}$, Eduardo M. Rego ${ }^{2,3}$, \\ Jan Jacob Schuringa (10) ${ }^{1}$ and Antonio R. Lucena-Araujo (iD) ${ }^{4}$
}

\begin{abstract}
Although the mixed lineage leukemia 5 (MLL5) gene has prognostic implications in acute promyelocyte leukemia (APL), the underlying mechanism remains to be elucidated. Here, we demonstrate the critical role exerted by MLL5 in APL regarding cell proliferation and resistance to drug-induced apoptosis, through mtROS regulation. Additionally, MLL5 overexpression increased the responsiveness of APL leukemic cells to all-trans retinoic acid (ATRA)-induced differentiation, via regulation of the epigenetic modifiers SETD7 and LSD1. In silico analysis indicated that APL blasts with MLL5 ${ }^{\text {high }}$ transcript levels were associated with retinoic acid binding and downstream signaling, while MLL5 ${ }^{\text {low }}$ blasts displayed decreased expression of epigenetic modifiers (such as KMT2C, PHF8 and ARID4A). Finally, APL xenograft transplants demonstrated improved engraftment of MLL5-expressing cells and increased myeloid differentiation over time. Concordantly, evaluation of engrafted blasts revealed increased responsiveness of MLL5-expressing cells to ATRA-induced granulocytic differentiation. Together, we describe the epigenetic changes triggered by the interaction of MLL5 and ATRA resulting in enhanced granulocytic differentiation.
\end{abstract}

\section{Introduction}

The mixed lineage leukemia 5 (MLL5) gene, also known as the lysine N-methyltransferase 2 (KMT2E), was originally proposed to be a functional member of the MLL family of proteins due to domain similarities ${ }^{1}$, despite of functional $^{2,3}$ and structural $^{4}$ disparities. Nevertheless, MLL5 interacts with the chromatin via its plant homeodomain (PHD) zinc finger to bind to the trimethylation of lysine 4 of histone 3 (H3K4me3) via a noncanonical

\footnotetext{
Correspondence: Antonio R. Lucena-Araujo (araujoarl@gmail.com)

${ }^{1}$ Department of Hematology, Cancer Research Centre Groningen, University Medical Centre Groningen, University of Groningen, Groningen, The Netherlands

${ }^{2}$ Center for Cell-Based Therapy, University of Sao Paulo, Ribeirao Preto, Sao Paulo, Brazil

Full list of author information is available at the end of the article These authors contributed equally: Diego A. Pereira-Martins, Isabel Weinhäuser These authors agreed to share the supervision of the study: Eduardo M. Rego, Jan Jacob Schuringa, Antonio R. Lucena-Araujo

Edited by $M$. Herold
}

binding mechanism ${ }^{5}$. In the hematopoietic tissue, MLL5 has been implicated to be involved in terminal myeloid differentiation, hematopoietic stem cell self-renewal ${ }^{6}$, and retinoic acid-induced granulopoiesis of normal human promyelocytes ${ }^{7}$. Yet, little is known about its pathophysiological importance in hematological malignancies.

In the clinical setting, previous studies have demonstrated that altered MLL5 transcript levels have prognostic implications in acute myeloid leukemia (AML) ${ }^{8}$ and acute promyelocytic leukemia (APL) $)^{9}$. In AML, the functional effect of MLL5 overexpression was only described regarding increased therapeutic response to decitabine, a DNA hypomethylating agent ${ }^{10}$, resulting in terminal differentiation. In the context of APL, the effects of MLL5 regarding the differentiation therapeutic agent (all-trans retinoic acid, ATRA), and its effect on disease burden and therapy response remains unclear. Here, we find that MLL5 overexpression led to enhanced APL

\section{(c) The Author(s) 2021}

\footnotetext{
(c) Open Access This article is licensed under a Creative Commons Attribution 4.0 International License, which permits use, sharing, adaptation, distribution and reproduction cc) in any medium or format, as long as you give appropriate credit to the original author(s) and the source, provide a link to the Creative Commons license, and indicate if changes were made. The images or other third party material in this article are included in the article's Creative Commons license, unless indicated otherwise in a credit line to the material. If material is not included in the article's Creative Commons license and your intended use is not permitted by statutory regulation or exceeds the permitted use, you will need to obtain permission directly from the copyright holder. To view a copy of this license, visit http://creativecommons.org/licenses/by/4.0/.
} 
response to all-trans retinoic acid (ATRA) and promoted primary APL engraftment with increased granulocytic differentiation in a xenograft model.

\section{Material and methods}

Lentiviral vectors and lentivirus production

Recombinant lentivirus encoding the PML-RARA fusion transcript (bcr1 isoform) and the HsMLL5 $\alpha$ gene (hereinafter called MLL5 gene) were generated using pCDH1-MCS1-EF1-GFP-Puro (pMEG) lentivector (\#CD713B-1; System Biosciences, USA) in HEK293T cells according to the three-plasmid packaging procedure as described elsewhere ${ }^{11}$. The complete optimized sequence of MLL5 gene was synthesized and fully sequenced in both directions by GenScript (Piscataway Township, USA). Lentiviral particles to overexpress $M L L 5$ gene were used to infect primary human leukemic blasts, NB4 and NB4-R2 cell lines. Particles containing the PML-RARA gene were used to transduce the U937 cells. Cells were sorted based on their GFP protein expression, and posteriorly used for in vitro assays. The efficiency of infection was further confirmed by gene expression quantification and western blotting analysis (Supplementary Fig. 1). For MLL5 knockdown, two sequences for shMLL5 (shMLL5\#1 - TRCN0000154711 and shMLL5\#2 TRCN0000358557; Sigma-Aldrich) vectors were properly expanded and validated as previously described ${ }^{12}$. A shRNA sequence that does not target human genes (referred to as scrambled) was used as a control (hereinafter called shCTRL).

\section{Cell lines and drugs}

All cell cultures were maintained in a humidified atmosphere at $37^{\circ} \mathrm{C}$ with $5 \% \mathrm{CO}_{2}$. NB4 (all-trans retinoic acid, ATRA-sensitive) and NB4-R2 (ATRA-resistant) cell lines were kindly provided by Dr. Pier Paolo Pandolfi (Harvard Medical School, USA), and maintained in RPMI 1640 (Gibco, USA) supplemented with 10\% fetal bovine serum (FBS) (Gibco, USA), L-glutamine (2 mM), 1\% penicillin/streptomycin (Invitrogen, USA). Mycoplasma contamination was routinely tested. All leukemia cell lines were authenticated by short tandem repeat analysis. The HEK293T (CRL-3216), U937 (CRL 1593-2 ${ }^{\mathrm{TM}}$ ) and HS27A (CRL-2496) cell lines were obtained from the American Type Culture Collection and grown in DMEM (for HEK293T and HS27A; Gibco, USA) or RPMI (for U937; Gibco, USA) with 10\% FBS. All-trans retinoic acid (ATRA) and arsenic trioxide (ATO) were obtained from SigmaAldrich (St. Louis, USA) and cytarabine (citarax) was obtained from Blau pharmaceuticals (Sao Paulo, Brazil).

\section{Patient samples}

Bone marrow samples of APL patients were studied after informed consent and protocol approval by the
Ethical Committee in accordance with the Declaration of Helsinki (registry \#12920; process number \#13496/2005; CAAE: 155.0.004.000-05). Mononuclear cells (MNCs) were isolated via Ficoll (Sigma-Aldrich) separation and cryopreserved.

\section{Gene set enrichment analysis (GSEA) for MLL5 biological pathways in APL}

Gene set enrichment analysis (GSEA) was performed using the Broad Institute software (http://software. broadinstitute.org/gsea/index.jsp). All genes from the RNA-seq of the TCGA AML cohort were pre-ranked according to their differential expression (fold change) and APL patients $(n=16)$ were categorized into high and low expression of $M L L 5$, using their median expression rate as a cut-off. Enrichment scores (ES) were obtained with the Kolmogorov-Smirnov statistic, tested for significance using 1000 permutations, and normalized (NES) to consider the size of each gene set. As suggested by the GSEA, a false discovery rate (FDR) cut-off of 25\% (FDR $q$ value $<0.25$ ) was used ${ }^{13}$. The limma-voom tool (http:// usegalaxy.org) was used to examine differentially expressed genes and genes with $\geq 1 \log$ difference and adjusted $p$ value of $<0.05$ were considered significant ${ }^{14}$. Data visualization was performed with the ClustVis platform ${ }^{15}$.

\section{In vitro primary APL co-cultures and ex vivo cultures}

HS27A cells were plated on gelatine coated culture flasks and expanded to form a confluent layer. Next, APL cells were cultured in Gartner's medium consisting of $\alpha$ MEM (Thermo Scientific) supplemented with $12.5 \%$ fetal bovine serum (Gibco), 12.5\% horse serum (Gibco), 1\% penicillin and streptomycin, $2 \mathrm{mM}$ glutamine (Gibco), $57.2 \mathrm{mM} \beta$-mercaptoethanol (Merck Sharp \& Dohme BV), $1 \mathrm{mM}$ hydrocortisone (Sigma-Aldrich) and $20 \mathrm{ng} / \mathrm{mL}$ G-SCF, TPO and IL-3. Co-cultures were grown at $37^{\circ} \mathrm{C}$ and $5 \% \mathrm{CO} 2$ and demi-populated weekly for counting. To evaluate the cell proliferation, the GFP content was measured at day 0 in $1 \times 10^{5}$ primary APL transduced cells (transduction efficiency) and co-cultured with HS27A cells for 14 days. The percentage of GFP positive $\left(\mathrm{GFP}^{+}\right)$cells was subsequently analyzed at day 7 and day 14. An increase in $\mathrm{GFP}^{+}$cells was interpretated as cell proliferation of the transduced cells. Granulocytic differentiation was evaluated in primary co-cultures after 8 days of ATRA treatment $(1 \mu \mathrm{M})$. At the end, cells were analyzed by FACS and histological evaluation was performed by May-Grünwald-Giemsa (MGG) staining. Images of MGG stained cells were made with a DM3000 (Leica) microscope.

\section{Western blot analysis}

Equal amounts of protein were used as total extracts, followed by SDS-PAGE, Western blot analysis with the 
indicated antibodies. For imaging the SuperSignal ${ }^{\mathrm{TM}}$ West Dura Extended Duration Substrate System (Thermo Fisher Scientific, USA) and Gel Doc XR + system (BioRad, Hercules, CA, USA) were used. Antibodies against CDKN1A (sc-71811), MLL5 (sc-377182), OP18 (sc55531), and $\alpha$-tubulin (sc-5286) were obtained from Santa Cruz Biotechnology (San Jose, CA). Antibodies against H3K4me3 (\#9727) and fibrillarin (\#2639) were obtained from Cell Signaling Technology (Danvers, USA). All membranes were incubated with a primary antibody following manufacturer's instructions (see Supplementary Table S1). Band intensities of cropped gels, which retained important bands were measured by the UN-SCAN-IT gel 6.1 software (Silk Scientific; USA).

\section{Quantitative real-time PCR}

Total RNA from U937 (transduced with control and PML-RARA), NB4 and NB4-R2 at basal conditions (transfected with control [empty vector and scrambled] or transduced with the MLL5 vectors [overexpression and silence]) or treated with ATRA $(1 \mu \mathrm{M})$ at different timepoints was obtained using the Trizol reagent (Thermo Fisher Scientific, USA). The cDNA was synthesized from $1 \mu \mathrm{g}$ of RNA using High-Capacity cDNA Reverse Transcription Kit (Thermo Fisher Scientific). Following total RNA extraction, real-time quantitative polymerase chain reaction (RQ-PCR) assays with sample-derived cDNA were performed in duplicate on MicroAmp optical 96well plates using a 7500 Real-Time PCR System (Applied BioSystems, USA) with the ACTB and GAPDH Standard Kit $(\# 4310881 \mathrm{E})$ as endogenous control. The gene expression of MLL5, SETD7 and SETD9 genes was determined using TaqMan Gene Expression Assay (MLL5: Hs00218773_m1, SETD7: Hs00363902_m1 and SETD9: Hs04187070_m1; Applied BioSystems), following the manufacturer's instructions. For LSD1, PHF8 and $R A R B$ quantification, the RQ-PCR was performed using Power SYBR Green Master Mix (Thermo Fisher Scientific) with specific primers for isoform A (referred LSD1A, forward: GTGGTAACAGGTCTTGGAGGG; reverse: CGTTGGCTTCATAAAGTGGGC), isoform b (referred $L S D 1 B$, forward: GACCGCCCTATGCAAGGAAT; reverse: GGGGGATTCGCTTCCAACTC), PHF8 (forward: GGGAAGAACCAACAACGCAG; reverse: TCTGGACGATAGCGCGG), RARB (forward: TCGGCACACTGCTCAATCAAT; reverse: TACACTCGAGGGGGAGGAAG), ACTB (Beta-actin, forward: AGGCCAACCGCAAGAAG; reverse: ACAGCCTGGATAGCAACGTACA) and GAPDH (GAPDH, forward: GGACTCATGACCACAGTCCAT; reverse: GCCATCACGCCACAGTTT) as endogenous control. The gene expression of the target genes was calculated relative to a reference cDNA (wild type NB4 cell line) and set to 1 . In all experiments, the same reference cDNA was used as an internal control to ensure that the results would be fully comparable among experiments. The gene expression values of the genes of interest were calculated as relative quantification using the $\Delta \mathrm{Ct}$ method and expressing the results as $2-{ }^{\Delta \Delta \mathrm{Ct}}$, in which $\Delta \Delta \mathrm{Ct}=\Delta \mathrm{Ct}_{\text {sample }}-\Delta \mathrm{Ct}_{\mathrm{NB} 4}$ or NB4-R2 cell line. All the results regarding the gene expression analysis, were normalized as a fold relative to wild-type NB4 cells. As a comparative control, we included the MLL5 gene expression evaluated in our own cohort of APL patients ${ }^{9}$, which was also normalized to the NB4 wild-type control (Supplemental Fig. 1C, D).

\section{Microarray and RNA-seq data analysis}

Microarray and RNA-seq data were obtained from the following datasets: GSE19201 ${ }^{16}$, GSE18397 ${ }^{17}$, and GSE12662 ${ }^{18}$, which were deposited in the GEO database (https://www.ncbi.nlm.nih.gov/geo). For the first two datasets, we investigated the mRNA expression of $M L L 5$, LSD1, SETD7, and SETD9 genes in samples from wild type NB4 cells, treated with vehicle (DMSO) and ATRA $(1 \mu \mathrm{M})$ at different time-points $(24,48$ and $72 \mathrm{~h})$. For the last dataset, we investigated the expression of the MLL5 gene in U937 cells transduced with an empty vector and Zinc-inducible promoter for the PML-RAR $\alpha$ gene, after 6 and $9 \mathrm{~h}$ of Zinc treatment. Genes that presented normalized counts equal to 0 for any replicates were excluded from the analysis.

\section{Chromatin immunoprecipitation analysis sequence (ChIP- seq)}

ChIP-seq data were obtained from the GSE18886 dataset $^{19}$, which was deposited in the GEO database (https://www.ncbi.nlm.nih.gov/geo). APL cell models (NB4 cells, primary APL blasts or PML-RARA transduced U937 cells) were treated $24 \mathrm{~h}$ with ATRA $(1 \mu \mathrm{M})$ and ChIP-seq was performed according to the previously described.

\section{In vitro assays \\ Cell proliferation}

Cells were treated with thymidine $(2 \mathrm{mM}$; CallBiochen, USA) for $18 \mathrm{~h}$ twice to induce cell cycle arrest at the G1/S boundary. Cells were subsequently seeded in 6-well plates and 1 million cells were collected and fixed with $70 \%$ ethanol at distinct timepoints: Day 1, 2, 3, 4, 6 and 8 and stored at $-20^{\circ} \mathrm{C}$. Ki-67 staining was performed following the manufacturer's instructions (Ki-67 PE clone SolA15; BioLegend, USA). Next, the mean of fluorescence intensity (MFI) was obtained by flow cytometry standard techniques using a FACSCalibur instrument (BectonDickinson, USA). IgG isotype was used as a negative control for each condition. As a confirmatory assay, we performed an MTT assay to measure cell viability. Transduced APL cell lines $\left(2 \times 10^{4}\right.$ cells/well $)$ were 
cultured in a 96-well plate in RPMI medium containing $10 \%$ FBS, during 1, 2, 4, 6 and 8 days. At the end of each culture period, $10 \mu \mathrm{L}$ of a $5 \mathrm{mg} / \mathrm{ml}$ solution of MTT was added to each well followed by an incubation of $4 \mathrm{~h}$ at $37^{\circ} \mathrm{C}$. The reaction was stopped using $100 \mu \mathrm{L}$ of $0.1 \mathrm{~N}$ $\mathrm{HCl}$ in anhydrous isopropanol. Cell viability was evaluated by measuring the absorbance at $570 \mathrm{~nm}$, using the $\mathrm{iMark}^{\mathrm{TM}}$ Microplate Absorbance Reader (Bio-Rad, Richmond, CA, USA). In parallel, cells were seeded at a density of $1 \times 10^{4}$ cells $/ \mathrm{ml}$ in $10-\mathrm{cm}$ dishes and the cell number was counted daily for seven days.

\section{Cell cycle analysis}

Cell cycle phases were determined by BD Cycletest ${ }^{\mathrm{TM}}$ Plus DNA Reagent Kit (Becton-Dickinson) according to the manufacturer's instructions. A total of $4 \times 10^{5}$ synchronized cells at G1/S phase were seeded in 24-well plates and collected and fixed at distinct timepoints: 0, 6, 8,10 and $12 \mathrm{~h}$. DNA content distribution was acquired with the FACSCalibur cytometer (Becton-Dickinson) and analyzed with the FlowJo software (Treestar, Inc., USA). Data from $12 \mathrm{~h}$ was used to generate the plots in Fig. $1 \mathrm{C}$.

\section{Apoptosis assay}

For the apoptosis analysis, $5 \times 10^{5}$ cells were seeded in 24-well plates and incubated in complete medium for $24 \mathrm{~h}$ in the presence of vehicle, ATO $(1 \mu \mathrm{M}$, alone or in combination with $1 \mu \mathrm{M}$ of ATRA) and cytarabine (Ara-C, $10 \mathrm{nM})$. The apoptosis rate was determined using the Annexin V-APC and propidium iodide (PI) binding assay (BD Biosciences, San Jose, CA, USA). All specimens were acquired by flow cytometry (FACSCanto; Becton-Dickison) and analyzed with the Flowjo software (Treestar, Inc., USA). All experiments were performed in triplicate and for each sample a minimum of 10000 events were acquired.

\section{Assessment of mitochondrial reactive oxygen species (mtROS) production}

For this purpose, $1 \times 10^{6}$ cells were treated with ATO $(1 \mu \mathrm{M})$ for $12 \mathrm{~h}$. Following the manufacturer's instructions mtROS levels were determined with the fluorogenic MitoSOX RedTM (Thermo Fisher Scientific) via flow cytometry procedures (FACSCalibur, Becton-Dickinson) and analyzed with the FlowJo software (Treestar, Inc.). Hydrogen peroxide $(0.1 \mu \mathrm{M})$ was used as a positive control for the experiment.

\section{Granulocytic differentiation induction}

$2 \times 10^{5}$ transduced NB4 and NB4-R2 cells were cultured in RPMI medium containing $10 \% \mathrm{FBS}$ in the presence of ATRA $(1 \mu \mathrm{M})$ alone or in combination with ATO $(1 \mu \mathrm{M})$ for 48 and $72 \mathrm{~h}$ at $37^{\circ} \mathrm{C}$ (DMSO and $\mathrm{NaOH}$ were used as vehicle control for ATRA and ATO, respectively). The differentiation rate was determined by evaluating the number of CD11b-, CD11c-, CD15-, and CD16-positive cells (percentage and MFI levels). Experiments were conducted using the FACS LSRII flow cytometer (BectonDickinson) and analyzed with the FlowJo software (Treestar, Inc.). In parallel, cytospin preparations stained with May-Grünwald-Giemsa (MGG) were used to evaluate morphological changes in NB4 cells treated with ATRA $(1 \mu \mathrm{M})$ for 5 days (compared to DMSO control).

\section{Colony formation assay}

Colony formation capacity was evaluated out in semisolid methylcellulose medium $\left(1.5 \times 10^{3}\right.$ cells $/ \mathrm{ml}$; MethoCult 4230; StemCell Technologies Inc., Canada). Clonogenic assays were performed in the presence of medium alone (without serum), vehicle (DMSO control $0.01 \%)$ and ATRA $(0.1$ and $0.5 \mu \mathrm{M})$. Colonies were detected after 10 days of culture by adding $1 \mathrm{mg} / \mathrm{ml}$ of MTT reagent and scored with the Image J quantification software (US National Institutes of Health, USA).

\section{In vivo APL xenotransplant}

All animals were housed under specific pathogen free conditions in individually ventilated cages during the whole experiment. The animals were maintained according to the Guide for Care and Use of Laboratory Animals of the National Research Council, USA, and to the National Council of Animal Experiment Control recommendations.

Eight week old female NSGS (NOD.Cg-Prkdcscid Il2rgtm1Wjl Tg(CMV-IL3,CSF2,KITLG)1Eav/MloySzJ) mice were purchased from the Jackson Laboratory. Mouse experiments were performed in accordance with the national and institutional guidelines, and all experiments were approved by the Institutional Animal Care and Use Committee of the University of Sao Paulo (IACUC-USP; Process number \#067/2018). Mononuclear cells were isolated from six different APL patients (each one isolated from a different APL patient; clinical characteristics in Supplemental Table S2) were depleted for $\mathrm{CD}^{+}$cells and transduced twice with empty vector or MLL5 vector (multiplicity of infection, MOI $>50$ ) using Retronectincoated plates (Takara). Forty-eight hours post transduction, GFP levels were checked by FACS (empty vector (mean \pm Standard Deviation): $20.5 \pm 2.5 \%$ and MLL5: $4.8 \pm 1.9 \%)$ and $1 \times 10^{6}$ transduced cells were directly injected into the tibia of the animals $(n=6$ for each group). Human $\mathrm{CD} 45^{+}$levels were measured regularly in blood obtained by sub-mandibular bleeding and mice were sacrificed after engraftment was confirmed (on a set time point of 12 weeks). Cells from the mouse organs including BM, spleen and liver were isolated and analyzed for the presence of human $\mathrm{CD} 45^{+}$cells, regardless of GFP expression (to analyze the transduced and non- 
A.

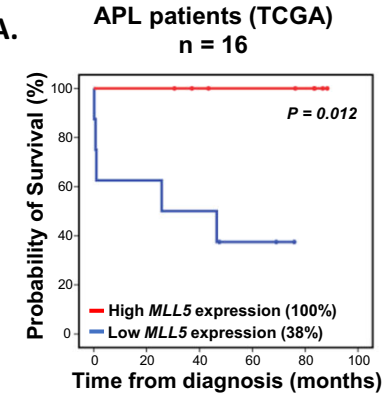

B. APL patients (TCGA)

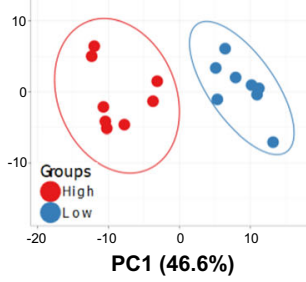

C.

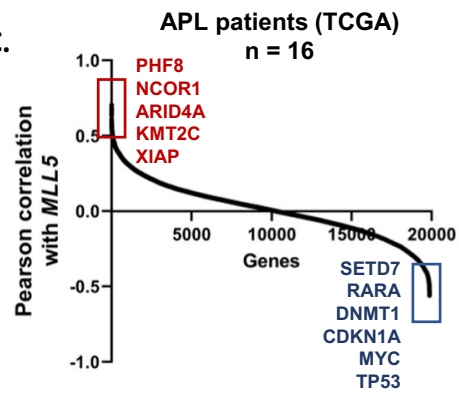

D. APL patients - TCGA cohort $\rightarrow$ RNA-seq (16 patients)

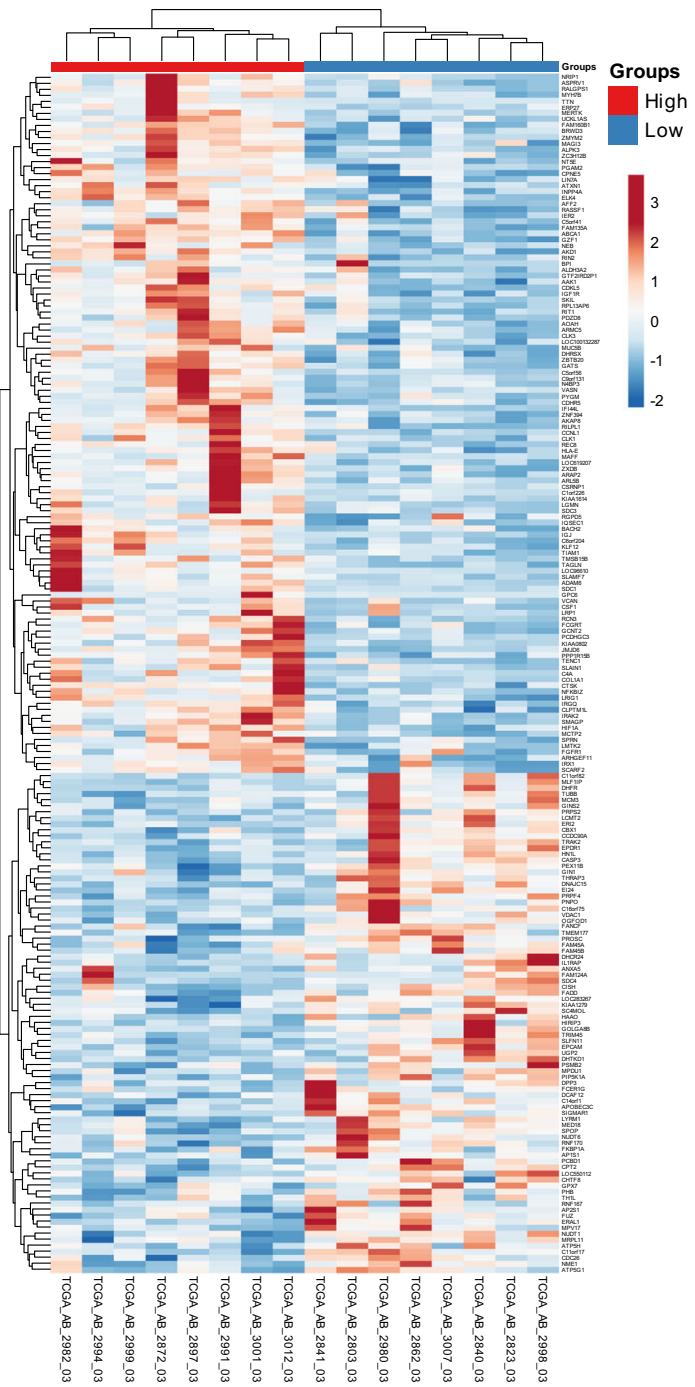

E.

GO_MYELOID_LEUKOCYTE_ GO_NEGATIVE_REGULATION DIFFERENTIATION
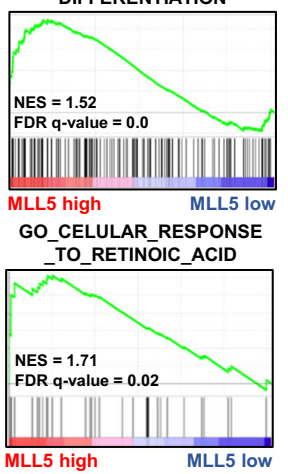

GO RETINOIC ACID BINDING

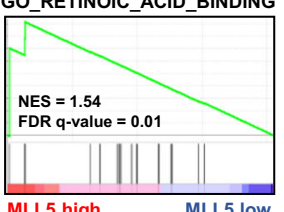

MLL5 high

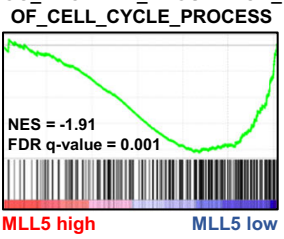

17_LSC (Dick et al., 2018)

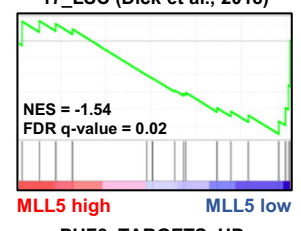

PHF8 TARGETS UP

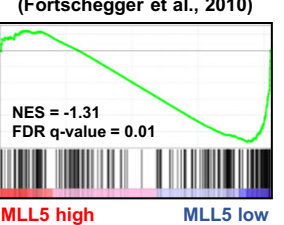

Fig. 1 Differential expression of MLL5 defines different biology in APL patients. The probability of overall survival in patients with APL included in the TCGA study according to MLL5 gene expression (A). Survival curves were estimated using Kaplan-Meier method, and the log-rank test was used for comparison. B Principal component analysis showing the differences in gene expression for APL patients included in the TCGA cohort dichotomized by MLL5 expression (high and low expression). Pearson correlation shows the positively and negatively correlated genes with MLL5 gene in APL patients (TCGA cohort). C Heatmap of differentially expressed genes (supervised clustering) in APL samples from TCGA cohort dichotomized according MLL5 gene expression (dichotomization point: median value; groups: high and low expression; $n=8$ in each group). D Gene set enrichment analysis (GSEA, lower panels) on a ranked gene list based on the leading-edge genes for MLL5 expression in 16 de novo APL patient samples from TCGA study. Genes were ranked based on Pearson correlation with MLL5 gene expression. Normalized enrichment score (NES) and false discovery rate (FDR) was used for significance. 
transduced human cells). Using FACS analysis, we evaluated the presence of human APL blasts and the more differentiated myeloid committed cells defined by the expression of $\mathrm{CD}_{4} 5^{+} \mathrm{CD} 117^{+} \mathrm{CD} 33^{+} \mathrm{HLADR}^{-} \mathrm{CD} 11 \mathrm{~b}^{-}$ $\mathrm{CD} 19^{-}$and $\mathrm{CD} 45^{+} \mathrm{CD} 117^{-} \mathrm{CD} 33^{+} \mathrm{CD} 11 \mathrm{~b}^{+}$markers respectively ${ }^{20}$, inside the $\mathrm{GFP}^{-}$(non-transduced) and $\mathrm{GFP}^{+}$(transduced cells) cell populations. All antibodies used for the staining were incubated following manufacturer's instructions (see Supplementary Table S1). Left over cells from BM were sorted for human APL blasts: $\mathrm{GFP}^{+} \mathrm{CD} 45^{+} \mathrm{CD} 117^{+} \mathrm{CD} 33^{+}$cells, to perform ex vivo cultures and stored in liquid nitrogen.

\section{Statistical analysis}

According to survival receiver operating characteristic (ROC) curve analysis, the median value of MLL5 expression was used to dichotomize patients into two groups (i.e., low expression, <4910; high expression, 24910 ). Overall survival was defined as the time from diagnosis to death from any cause; those alive or lost to follow-up were censored at the date last known alive. For patients who achieved CR, DFS was defined as the time from $C R$ achievement to the first adverse event: relapse, development of secondary malignancy, or death from any cause, whichever occurred first. All P values were two sided with a significance level of 0.05 . All statistical analyses were performed using the statistical package for the social sciences (SPSS) 19.0 and R 3.3.2 (The CRAN project, www.r-project.org) software.

\section{Results}

\section{MLL5 expression correlates with enhanced retinoic acid} response in APL patients

Increased MLL5 expression was reported to be associated with improved prognosis in APL when treated with ATRA plus anthracycline-based chemotherapy ${ }^{9}$. To validate our findings, we analyzed the $M L L 5$ gene expression in APL patients, using the TCGA public dataset ${ }^{21}$. Our analysis indicated increased overall survival in APL patients with high MLL5 expression (Fig. 1A). Principle component analysis using the transcriptome of APL patients (included in the TCGA cohort) demonstrated distinguished gene expression signature between the two groups (high $M L L 5$ expression vs Low $M L L 5$ expression) (Fig. 1B). Interestingly, APL patients with high $M L L 5$ expression were associated with increased expression of epigenetic modifiers (KMT2C, PHF8, and ARID4A) (Fig. $1 C$, D). Subsequent, gene set enrichment analysis (GSEA) associated MLL5 ${ }^{\text {high }}$ APL patients with the terms "Retinoic acid binding", "Myeloid leukocyte differentiation" and "Cellular response to retinoic acid", while low MLL5 APL patients were associated with "negative regulation of cell cycle", "17-leukemic stem cell markers" and "genes up-regulated upon PHF8 knockdown" (Fig. 1E). As a next step, we decided to evaluate the function of high and low MLL5 expression in vitro using APL cell lines.

\section{MLL5 significantly impacts on APL cell proliferation and cell cycle progression}

The human coding sequence of the MLL5 gene was stably overexpressed in NB4 and NB4-R2 cell lines. To confirm the overexpression of MLL5 at the protein level, protein lysates from transduced cell lines were prepared according to the described methods. As shown in Supplemental Fig. 1A, B, cells infected with pMEG-MLL5 and shMLL5 lentivirus efficiently overexpressed and downregulated the MLL5 protein (in comparison with the Empty vector and shScrambled, respectively). The level of $M L L 5$ expression post transduction was compared to the level of MLL5 detected in APL patients in a previous study ${ }^{9}$. As depicted by supplemental Fig. 1C, D, the overexpression and knockdown of MLL5 in APL cell lines corresponded to patients with high and low MLL5 expression, respectively.

Daily cell count of NB4 and NB4-R2 cell lines overexpressing MLL5 indicated increased cell proliferation (Fig. 2A), which was further confirmed by an MTT assay (Supplemental Fig. 2A) and Ki-67 staining (Fig. 2B). Furthermore, MLL5 overexpression resulted in accumulation of cells in the G2/M phase and enhanced colony formation capacity (Fig. 2C, D). Conversely, these effects were abrogated upon short hairpin knockdown of MLL5 (Supplemental Fig. 2B-E). In summary our data suggests that MLL5 promotes APL cell proliferation and colony formation by accelerating cell cycle progression.

MLL5 overexpression results in increased APL cell viability and resistance to drug induced apoptosis through $\mathrm{mtROS}$ regulation

Next, we treated the transduced APL cell lines with arsenic trioxide (ATO), ATO + ATRA and cytarabine (Ara-C) for 24, 48 and $72 \mathrm{~h}$. Overexpression (Fig. 2E) and knockdown (Supplemental Fig. 3A, B) of MLL5 led to increased resistance to drug-induced apoptosis in a timedependent manner.

Studies reported that the encoded MLL5 protein modulates intracellular reactive oxygen species (ROS) levels ${ }^{22}$. Given, that ATO induces ROS accumulation ${ }^{23}$ we hypothesized that MLL5 overexpression affects ATO induced mitochondrial ROS (mtROS) generation. Our analysis indicated a 3-fold decrease of mtROS levels in MLL5transduced and ATO stimulated cells compared to control (Fig. 2F), but no difference in shMLL5 cells (Supplemental Fig. 3C). Altogether, these findings demonstrate that MLL5 overexpression endows APL cells with a growth advantage and enhanced ROS clearance, which culminates in increased resistance to arsenic/anthracycline drug-induced apoptosis. 


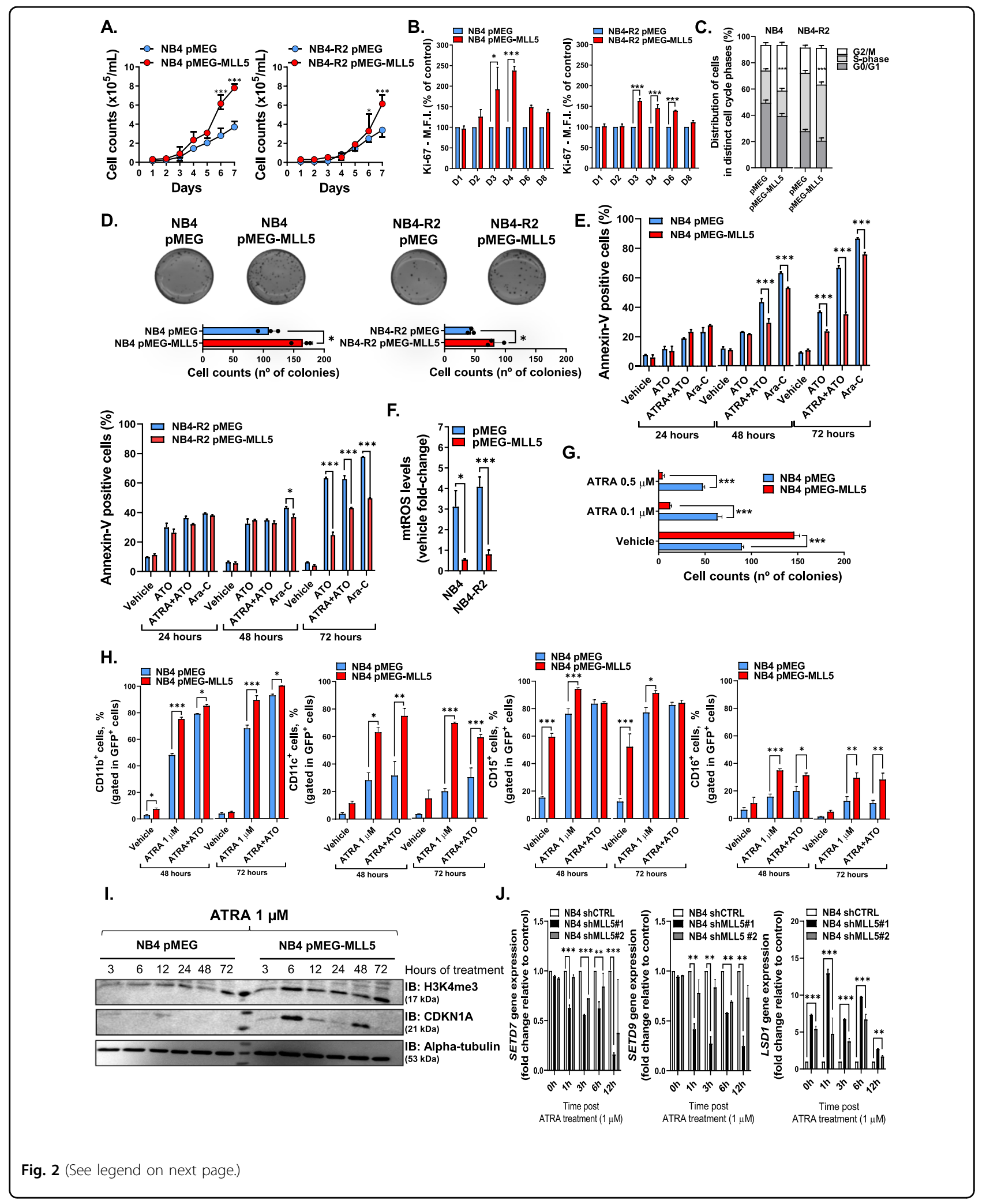




\begin{abstract}
(see figure on previous page)
Fig. 2 Effect of MLL5 on proliferation assays. A Growth curves, B Ki67 staining and C cell cycle analysis of NB4 and NB4-R2 cell lines lentivirally transduced with MLL5 or empty vector (pMEG, control). D Representative example of one of four independent experiments of colony formation assay in methylcellulose using lentivirally transduced NB4 and NB4-R2 cell lines. Graphic bars represent the number of colony-forming cells in each well. E Graphic bars represent the number of colony-forming cells in each well in NB4 cells transduced with MLL5 or empty vector (pMEG, control) treated with vehicle (DMSO, control) and ATRA (0.1 and $0.5 \mu \mathrm{M})$. Effect of MLL5 on drug-induced apoptosis assay. F Percentage of apoptotic cells after 24,48 and $72 \mathrm{~h}$ in culture with apoptotic stimulus (ATRA $1 \mu \mathrm{M}$; ATO $1 \mu \mathrm{M}$ and AraC $10 \mathrm{nM}$ ). G Mitochondrial reactive oxygen species (mtROS, MitoSOX'M $5 \mu \mathrm{M})$ measured by FACS in lentivirally transduced cell lines after $12 \mathrm{~h}$ of treatment with ATO $(1 \mu \mathrm{M})$. All experiments were performed in triplicate. Effect of MLL5 on myeloid differentiation. $\mathbf{H}$ Percentage of CD11b $\mathrm{b}^{+}, \mathrm{CD} 11 \mathrm{c}^{+}, \mathrm{CD} 15^{+}$and $\mathrm{CD} 16^{+}$in NB4 cells infected with empty vector or pMEGMLL5 lentiviruses after 48 (left panel) and 72 (right panel) h of ATRA $(1 \mu \mathrm{M})$ alone or in combination with ATO $(1 \mu \mathrm{M}$ each) treatment used as the standard stimulus for differentiation. Effect of MLL5 knockdown on myeloid differentiation. I Western blot for H3K4me3, CDKN1A (p21) of total protein lysates from NB4 cell line infected with empty vector or PMEG-MLL5 lentiviruses and treated with ATRA $1 \mu M$. A total of $30 \mu g$ of protein was loaded in each lane. J Gene expression analysis of SETD7, SETD9 and LSD1 in NB4 cell line infected with shRNAs targeting MLL5 (shMLL5) and scrambled controls (shCTRL) upon ATRA treatment $(1 \mu \mathrm{M}$ ). The expression of SETD7, SETD9 and LSD1 genes was quantified by Real-time quantitative PCR (RQ$P(R)$ using GAPDH and $A C T B$ as endogenous control. Note: Data from continuous variable were all expressed as mean \pm standard error of the mean. ${ }^{*} P<0.05$. ${ }^{* *} P<0.001$. NS indicates not significant.
\end{abstract}

\section{MLL5 sensitizes APL cells to ATRA-induced granulocytic differentiation via the recruitment of an epigenetic machinery}

Earlier publications revealed that MLL5 is implicated in terminal myeloid and retinoic acid-induced differentiation ${ }^{6}$. Given that ATRA is the standard therapeutic agent in APL, we decided to investigate how MLL5 affects ATRA-induced myeloid differentiation in APL, using the ATRA responsive NB4 cell line. Interestingly, while the overexpression of MLL5 drives proliferation and clonogenicity (Fig. 2A-D), we noticed a significant reduction of colonies in MLL5 overexpressing cells upon ATRA stimulation (Fig. 2G). To evaluate differentiation, we analyzed the expression of the granulocytic/monocytic differentiation markers CD11b, CD11c, CD15 and CD16 in NB4-MLL5 cells at basal conditions and when treated with ATRA (alone or in combination with ATO, $1 \mu \mathrm{M}$ ) (Fig. 2H, Supplemental Fig. 4A). Intriguingly, CD11b and CD15 expression was increased in NB4-MLL5 cells at basal conditions, while the treatment with ATRA \pm ATO further enhanced the expression of all differentiation markers compared to control cells. In accordance with our FACS data, morphological cell evaluation revealed that NB4-MLL5 cells treated with ATRA $(1 \mu \mathrm{M}$, 5 days) exhibited a decreased nucleus-cytoplasm ratio with nuclear lobulation, suggestive of neutrophilic differentiation, which was not observed in control cells (Supplemental Fig. 4B). Moreover, also MLL5 transduced NB4-R2 cells presented increased expression of CD15 at basal conditions. Overall, NB4-R2-MLL5 cells displayed enhanced granulocytic differentiation after $48 \mathrm{~h}$ and $72 \mathrm{~h}$ of ATRA + ATO treatment (Supplemental Fig. 4C). These results suggest that the ATRA and ATO-mediated disruption of the PML-RARA fusion protein in APL cells plays an essential role in MLL5induced granulocytic differentiation.

Since PML-RARA recruits a repressive epigenetic complex, which dissociates upon ATRA treatment via PHF8 activation (causing H3K4 hypermethylation) ${ }^{24}$, we investigated the effect of MLL5 on trimethylation of H3K4 in response to ATRA. After $6 \mathrm{~h}$ of ATRA treatment $(1 \mu \mathrm{M})$, we observed a significant increase of the H3K4me3 and CDKN1A (Fig. 2I) in MLL5-expressing cells followed by an up-regulation of the histone-modifying enzyme SETD7 gene after $12 \mathrm{~h}$ of treatment (Supplemental 4D). PHF8 expression was significantly increased in NB4-MLL5 cells treated with ATRA alone after $72 \mathrm{~h}$, while these effects were observed already at $24 \mathrm{~h}$ when ATRA was combined with ATO (Supplemental Fig. 4E). As a result of this activation, the $R A R B$ expression (the main downstream target of PHF8, which promotes promyelocytic differentiation) was significantly up-regulated in MLL5-overexpressing cells upon ATRA and ATRA + ATO treatments (Supplemental Fig. 4F). Contrarily, shMLL5 cells exhibited a consistent downregulation of the SETD7 and SETD9 genes, while the LSD1 gene was up-regulated (Fig. 2J). To validate our findings, we evaluated the expression of MLL5 and its partners using non-transduced NB4 cells treated with ATRA $(1 \mu \mathrm{M})$ at different time-points. As expected, we detected an upregulation of the CDKN1A and MLL5 genes after 48 and $72 \mathrm{~h}$ of treatment (Fig. 3A). Additionally, upon ATRA treatment, NB4 cells presented a time-dependent downregulation of the $L S D 1$ gene expression but no difference for SETD7 and SETD9 genes (Fig. 3A). Using a zincinducible model for PML-RARA expression in U937 cells, we observed a reduction of MLL5 levels over time when PML-RARA expression was induced. These effects were abrogated by the administration of ATRA, which restored the transcript levels of MLL5 (Fig. 3B).

Next, we examined the MLL5 promoter region, to evaluate the main epigenetic marks in APL patients treated with ATRA and control (DMSO) ${ }^{25}$. We noticed that upon ATRA treatment, no changes were observed for H3K27me3 and H3K36me3 tracks, while only minor changes were observed for H3K4me3 and H3K27ac at the MLL5 locus (Fig. 3C). Subsequently, we evaluated the gene ontology (GO, biological process (BP)) process associated with the genes that had significant chromatin 


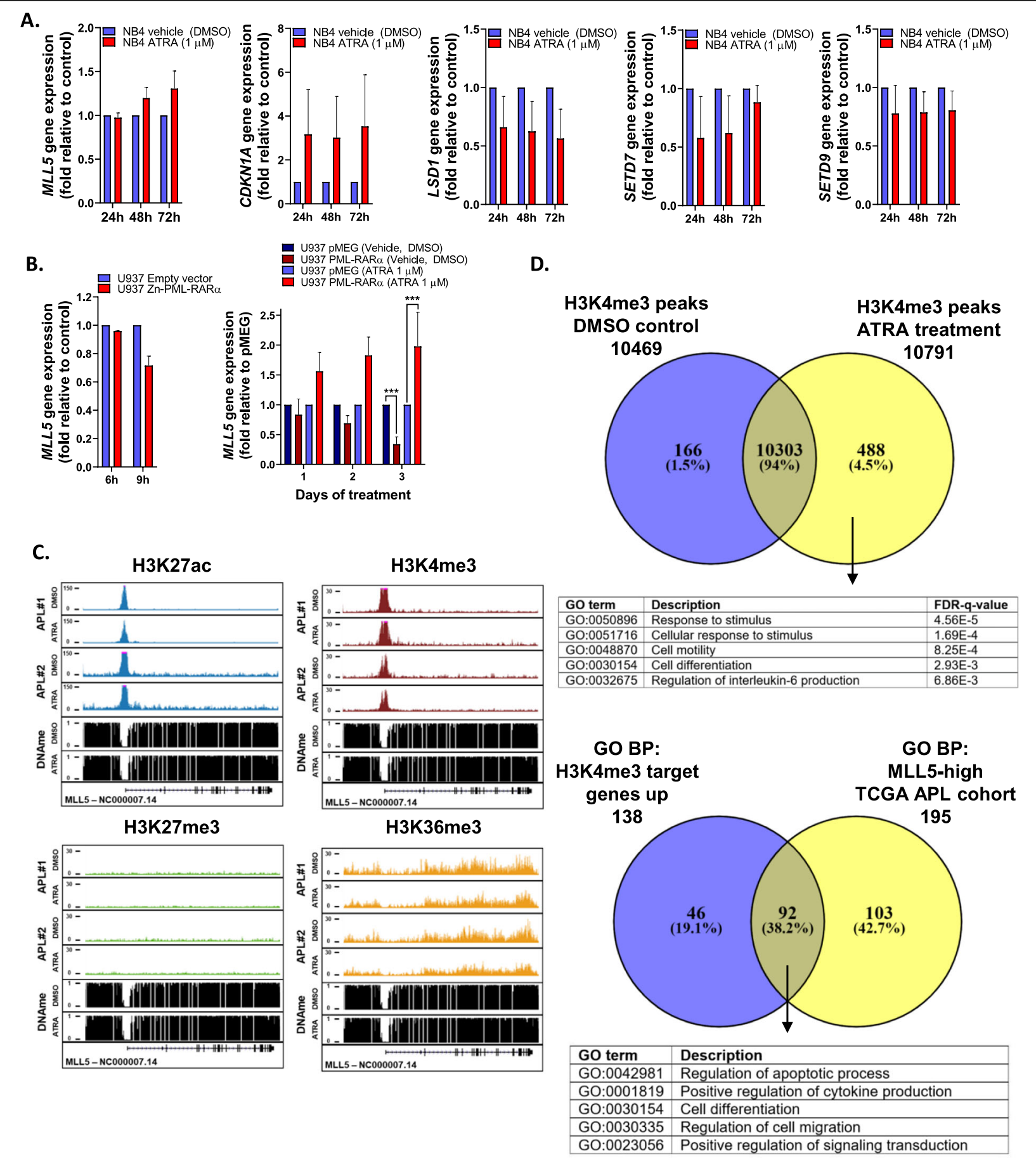

Fig. 3 Molecular effects of ATRA treatment in PML-RARa+ cells. A Gene expression analysis of MLL5, LSD1, SETD7, SETD9, and CDKN1A in NB4 cell line treated with vehicle (DMSO) and ATRA (1 HM) for 24, 48 and $72 \mathrm{~h}$. Expression data was retrieved from GSE19201 and GSE18397. B Gene expression analysis of MLL5 in U937 cell line infected with empty-vector and Zinc-inducible PML-RARa (left panel). The expression of MLL5 gene was quantified 6 and $9 \mathrm{~h}$ after Zinc administration. Gene expression analysis of MLL5 in U937 cell line infected with empty-vector and PML-RARa (bcr1 isoform; right panel). Cells were treated with ATRA $(1 \mu \mathrm{M})$ and DMSO control for 1, 2 and 3 days. Data were expressed as mean \pm standard error of the mean. C Overview of APL ChIP-seq (H3K27ac, H3K4me3, H3K27me3 and H3K36me3) data at the genomic regions of the MLL5 gene before and after ex vivo ATRA treatment. D Upper panel exhibit the overlap in H3K4me3-bound loci determined by ChIP seq in APL patient\#1. Peaks were distinguished in present only in DMSO control, only upon ATRA treatment and in both conditions. Overall, 488 genes were enriched only upon ATRA treatment, which were associated with 138 gene ontology (GO)_biological process (BP). Lower panel exhibit the GO BP for H3K4me3 up regulated genes present only upon ATRA-treatment and the GO BP found in APL patients from the TCGA cohort with high MLL5 expression. ChIP-seq data was retrieved from GSE18886. ${ }^{*} P<0.05$. ${ }^{* *} P<0.01 .{ }^{* * *} P<0.001$. NS indicates not significant. 
occupancy for H3K4me3 upon ATRA-treatment (since high MLL5 expression increased H3K4me3 in our in vitro model). A total of 488 genes were only present in the ATRA group (Fig. 3D, upper panel), which were associated with $138 \mathrm{GO}$ BP processes (Fig. 3D). Interestingly, comparing the GO BP signature using the APL patients included in the TCGA cohort with high MLL5 expression, we noticed a high overlap between the GO process (Fig. 3D, lower panel). Gene ontology analysis for terms present in both MLL5high APL patients and H3K4me3 ATRA only were associated with "Positive regulation of cytokine production", "cell differentiation" and "regulation of apoptotic process", suggesting that APL patients with high $M L L 5$ expression presented an intrinsic biology more compatible with ATRA-induced changes on H3K4me3 (Supplementary Table 3).

\section{Ex vivo evaluation of primary APL blast cells expressing MLL5 present similar phenotypes to APL cell lines}

Since APL cell lines (NB4 and NB4-R2 cells) exhibit aberrant karyotypes that are not commonly found in APL patients $^{26}$, we decided to study the role of MLL5 expression in primary APL blasts. Concordant with our cell line data, we observed increased differentiation upon ATRA treatment assessed by $\mathrm{CD} 11 \mathrm{~b}$ expression and cellular morphology (Fig. 4A-C). Morphological analysis of empty-vector (pMEG) and MLL5 transduced primary blasts treated with DMSO presented features of immature APL blasts, while ATRA treatment induced increased differentiation in MLL5-transduced cells (red arrows, Fig. 4A) compared to control. Additionally, we observed ex vivo clonal expansion of $\mathrm{GFP}^{+}$MLL5-transduced cells over time, while no difference was observed for the empty vector controls (Fig. 4D). Similar to our findings in APL cell lines, the MLL5 overexpression in primary APL blasts enhanced cell proliferation and potentiated the effect of ATRA induced differentiation.

\section{MLL5-expressing APL blasts promote increased engraftment in a xenograft model, associated with increased granulocytic differentiation}

Finally, we generated a xenograft mouse model using primary APL samples transduced with the PMEG-MLL5 or controls to assess the impact of MLL5 on APL cell viability and differentiation in vivo (Fig. 4E). At week 12, the engraftment of human APL cells was confirmed in both groups and the animals were sacrificed to analyze the level of human APL blast infiltration (transduced and nontransduced pMEG and pMEG-MLL5 cells) in bone marrow (BM), spleen, and liver. Interestingly, the BM of MLL5transplanted mice presented increased levels of human CD45 + cells, GFP expression and frequency of CD11b+ compared to control (Fig. 4F-H). This result suggests that MLL5 promoted expansion of the transduced cells $\left(\mathrm{GFP}^{+}\right)$ and enhanced myeloid differentiation, which was not observed in the $\mathrm{GFP}^{-}$population (Supplementary Fig. 5A). Furthermore, MLL5-transduced cells showed inferior engraftment compared to controls (Fig. 4H), reinforcing the idea that MLL5 induces APL cell proliferation (Supplementary Fig. 5C, first panel). No differences in engraftment/ weight were observed in the liver and spleen between MLL5 and control mice (Fig. 4I, J). Weekly hematological counts revealed no differences in peripheral blood leukocyte and platelet counts, while we observed a reduction in hemoglobin levels in empty vector mice at later time points (week 12) (Supplementary Fig. 5B).

To further study cellular characteristics of engrafted MLL5 cells, BM cells from empty vector and MLL5-transplanted mice were sorted (based on $\mathrm{GFP}^{+}$and human $\mathrm{CD} 45^{+} \mathrm{CD} 33^{+} \mathrm{CD} 117^{+}$expression) (supplementary Table 2 shows the MLL5 gene expression in the murine $\mathrm{BM}$ of sacrificed animals) for ex vivo experiments. Sorted $\mathrm{GFP}^{+}$ human APL blasts were cultured for 8 days with ATRA $(1 \mu \mathrm{M})$ and ATRA + ATO $(1 \mu \mathrm{M}$ each $)$ to determine induction of granulocytic differentiation or ATRA + ATO to evaluate drug induced apoptosis (since ATRA alone induces very low levels of apoptosis ${ }^{27}$ ). Xenograft derived ex vivo cultures, revealed that $M L L 5$-expressing cells presented decreased sensitivity to ATO induced apoptosis after $72 \mathrm{~h}$ of treatment and higher CD11b levels at day 8 of ATRA administration compared to control cells (Fig. 4L). Interestingly, the number of MLL5 transduced apoptotic cells was slightly increased (inferior to $10 \%$ ) when treated with the vehicle compared to pMEG cells. This could be attributed to the enhanced proliferative rate of MLL5 cells, which leads to a quicker exhaustion of nutrients. These results are in accordance with the previous in vitro and patient ex vivo observations, showing, that MLL5 enhances ATRA-induced differentiation in APL, which could be confirmed in a xenograft mouse model (Fig. 4M).

\section{Discussion}

Here, we demonstrated for the first time that increased MLL5 expression improves ATRA-driven therapeutic response in APL. Since, high MLL5 transcript levels were associated with favorable prognosis in APL patients treated with ATRA and anthracycline-based chemotherapy ${ }^{9}$, we hypothesized that this could emanate from a lower tumor burden or higher therapy response. However, when MLL5 was overexpressed in APL cells we observed increased proliferation and resistance to drug induced apoptosis. Contrarily, in the presence of ATRA the proliferation rate of MLL5 was severely impaired and concomitantly the granulocytic differentiation rate was enhanced. These effects were mediated via a sustained increase of $\mathrm{H} 3 \mathrm{~K} 4 \mathrm{me} 3$ across the genome and the modulation of histone-modifying enzyme-related genes. Interestingly, the modulation of histone-modifying enzymes was only observed in the context of MLL5 


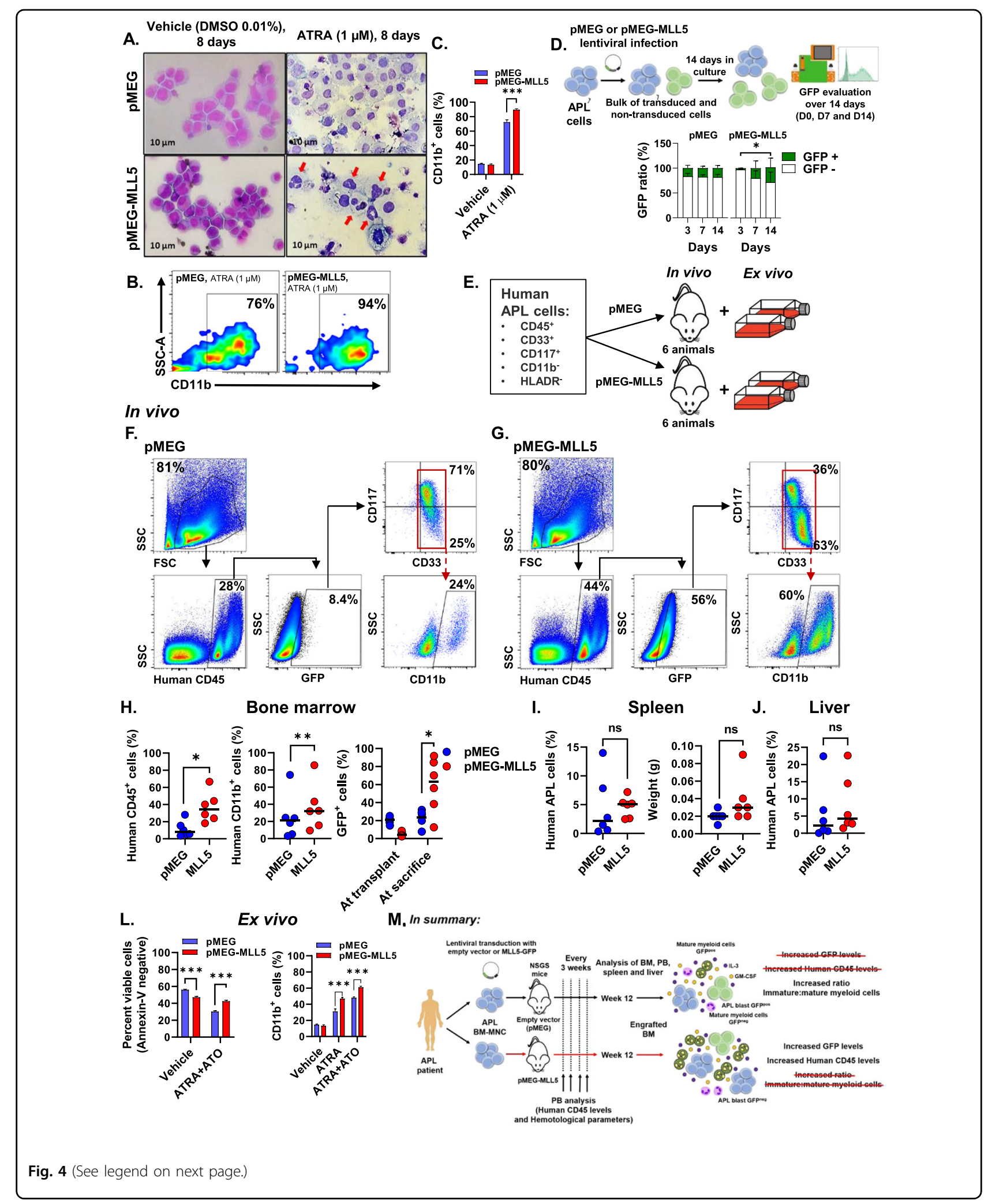

knockdown, but not when MLL5 was overexpressed. Moreover, the extent of the biological effect observed upon MLL5 silencing was correlated to the efficiency of the respective short hairpin to downregulate MLL5 expression. One reason why we could only observe a change in histonemodifying enzyme expression when MLL5 was silenced, 
(see figure on previous page)

Fig. 4 Primary MLL5 transduced APL blasts (GFP ${ }^{+}$cells) exhibited increased cell proliferation and granulocytic differentiation in vitro and in vivo. A Representative images of May-Grünwald-Giemsa-stained cytospins of transduced primary APL blasts (pMEG, and pMEG-MLL5) treated 8 days with ATRA $(1 \mu \mathrm{M})$ and DMSO control (vehicle, $0.01 \%)$. B Fluorescence-activated cell sorting (FACS) immunophenotype of CD11b staining of transduced APL blasts with MLL5 or empty vector and C percentage of CD $11 b^{+}$cells in transduced primary blasts after 8 days of ATRA (1 $\mu M$ ) treatment as the standard stimulus for differentiation. Data were expressed as mean \pm standard error of the mean. D Schematic representation of cell proliferation assay based on GFP expression in primary APL cells transduced with MLL5 and the empty vector control (pMEG). Graphic bars represent the number of GFP+ cells inside the bulk of transduced primary APL blasts during 14 days of culture. Data were expressed as mean \pm standard error of the mean. Overview of the mouse xenograft for APL. E Schematic representation of the generation of the xenograft mouse model for APL engraftment using NSGS mice. Representative FACS phenotype from a primary murine bone marrow transplanted with human transduced APL blasts with the empty vector $(\mathbf{F})$ or the MLL5 gene $(\mathbf{G})$ at sacrifice. APL blasts and mature myeloid committed cells were analyzed by flow cytometry using markers against CD117, CD33 and CD11b as indicated (inside the population huCD45 $5^{+}$and $\mathrm{GFP}^{+}$). Scatter plots showing engraftment of donor human $\mathrm{CD}_{4} 5^{+}$cells (regardless the GFP expression), human $\mathrm{CD} 45^{+} \mathrm{GFP}{ }^{+}$cells and human $\mathrm{CD} 45^{+} \mathrm{GFP}^{+} \mathrm{CD} 11 \mathrm{~b}^{+}$cells in bone marrow $(\mathbf{H}), \mathbf{I}$ spleen (percentual of engraftment and spleen weight) and $\mathbf{J}$ liver of transplanted mice at sacrifice. Data were expressed as median values. Ex vivo analysis of transduced APL blasts reinforces in vitro findings. $\mathbf{L}$ Incubation of bone marrow sorted APL blasts cells $\left(\mathrm{GFP} \mathrm{C}^{+} \mathrm{CD} 45^{+} \mathrm{CD} 117^{+} \mathrm{CD} 33^{+}\right)$from pMEG/MLL5 engrafted mice, with ATRA plus ATO ( $1 \mu \mathrm{M}$ each) led to increased induction of apoptosis over the course of $72 \mathrm{~h}$ in empty vector cells. Percentage of CD11 $b^{+}$cells in sorted blasts from murine BM isolated from engrafted pMEG and MLL5 mice, after 8 days of ATRA alone or in combination with ATO $(1 \mu \mathrm{M}$ each) treatment as the standard stimulus for differentiation. Data were expressed as mean \pm standard error of the mean. $\mathbf{M}$ Summary results from APL xenograft murine model to study the role of MLL5 in APL. ${ }^{*} P<0.05$. ${ }^{* *} P<0.01 .{ }^{* *} P<0.001$. NS indicates not significant.

could be because of the changes in interactomes when MLL5 is overexpressed ${ }^{10}$. To our knowledge, this is the first study to evaluate the interplay between MLL5 and SETD7/9 and LSD1, using both overexpression and knockdown models in APL. In line with previous publications, Sebastian et all $(2009)^{3}$, demonstrated the indirect effect of MLL5 on H3K4 trimethylation via the regulation of SETD7/9 and LSD1 in myoblasts, in the context of MLL5 knockdown cells. Similar results were reported by our group in AML cell lines in vitro and in vivo (Almeida et al, 2018). While our study appears to be in contradiction with previous reports at first, it is critical to mention that the function of MLL5 in AML was investigated in the context of epigenetic-targeted therapy. Hence, our and previous studies reinforce the idea that the function of MLL5 can be orchestrated by epigenetic changes and that the classification of MLL5 as a pro/anti-tumorigenic gene should be considered within context.

In addition, the reduction of MLL5 expression was previously associated with changes at the H3K4 but not H3K9 histone mark ${ }^{17,21}$. Despite the up regulation of H3K4me3 observed in vitro, APL patients presented only a modest increase for H3K4me3 and H3K27ac at the MLL5 locus upon ATRA treatment ${ }^{17,21}$. We also noticed that the biological process observed in APL patients with high MLL5 levels overlapped with genes harboring high H3K4me3 chromatin-occupancy upon ATRA treatment. These observations suggest that patients with high MLL5 levels exhibit similar genetic signatures as APL samples treated with ATRA. Considering cell cycle progression, Deng et al. reported that ectopic overexpression of MLL5 leads to cell cycle arrest in G1-phase ${ }^{28}$, while Cheng et al. observed cell cycle inhibition upon $M L L 5$ knockdown $^{29}$. It is not uncommon that overexpression or knockdown of the same gene, causes analogous biological effects, indicating tissue dependent functions for MLL5. Future studies should consider the use of inducible models for
MLL5 knockdown/overexpression to provide better comparability among the different phenotypes associated with MLL5 modulation in leukemic cells.

Furthermore, we observed increased resistance of MLL5 cells upon ATO and Ara-C treatment. Studies suggested that the loss of MLL5 leads to extensive DNA damage and elevated ROS levels ${ }^{22}$. Considering that ATO-mediated toxicity partly relies on ROS production to induce PML nuclear body formation and $\mathrm{p} 53$ pathway activation ${ }^{23}$, it is probable that the resistance of MLL5 overexpressing cells is linked to reduced mtROS generation.

Although, APL xenotransplants are extremely challenging, we managed to provide a model, which satisfactory recreates the disease to investigate APL genetic modifiers ${ }^{30}$. MLL5 overexpression promoted cell proliferation and improved human engraftment along with GFP expansion. Yet, when we analyzed the amount of APL blast cells (defined by $\mathrm{CD} 117^{+} \mathrm{CD} 33^{+}$) versus myeloid committed cells $\left(\mathrm{CD} 17^{-} \mathrm{CD}^{+}{ }^{+} \mathrm{CD} 1 \mathrm{~b}^{+}\right)$in the $\mathrm{GFP}^{+}$fraction, we observed a ratio (APL blasts: myeloid committed) of 1:2 in MLL5 transplanted mice compared to 3:1 in control mice. Given, that the interplay between MLL5 and ATRA facilitates differentiation in vitro, it is conceivable that MLL5 drives differentiation also in vivo due to, the long-term exposure (12 weeks) of high levels of hematopoietic cytokines, such as IL3 and GM-CSF ${ }^{31}$. However, these differences could be abrogated over time. Whether the altered expression of $M L L 5$ gene is cause or consequence of the malignant myeloid transformation process remains unclear. Nevertheless, in the context of ATRA based therapy, our data suggests that the categorization of APL patients based on MLL5 expression at diagnosis can have important prognostic values.

Our findings may be of particular interest if we consider epigenetic enzymes as bona fide targets for anti-AML drug development ${ }^{32}$. In this context, Schenk et al. demonstrated that inhibition of the LSD1 demethylase 
may reactivate the ATRA-differentiation pathway in nonAPL $\mathrm{AML}^{33}$. Although the authors did not investigate the interaction between LSD1 and MLL5, it is conceivable that MLL5 mediates or potentiates the inhibition of LSD1 in the presence of ATRA, and in part, modulates the epigenome to improve drug response. One may argue that such an approach may be irrelevant for APL in light of the well-succeed state-of-the-art combination of ATRA and $\mathrm{ATO}^{34-36}$. That was one of the reasons why we decided not to evaluate APL overall survival in vivo in the context of MLL5. Yet, we support the belief of transferring the experience gained from a well-characterized disease, such as APL, to another more challenging leukemia subset. As a result, likewise to our APL study, our group observed increased responsiveness to ATRA induced differentiation when AML cell lines were transduced with MLL5 in vitro, which also led to significant tumor burden reduction in vivo (Almeida et al., 2018). Our data indicates a relevant crosstalk between the ATRA-induced differentiation pathway and histone H3K4 methylation, which suggests that epigenetic enzymes may constitute a promising therapeutic target for both APL and non-APL AML.

\section{Acknowledgements}

We thank to Dr. João Agostinho Machado-Neto and Carolina Hassibe Thomé for the technical support.

\section{Author details \\ 'Department of Hematology, Cancer Research Centre Groningen, University Medical Centre Groningen, University of Groningen, Groningen, The Netherlands. ${ }^{2}$ Center for Cell-Based Therapy, University of Sao Paulo, Ribeirao Preto, Sao Paulo, Brazil. ' ${ }^{3}$ ematology Division, LIM31, Faculdade de Medicina, University of Sao Paulo, Sao Paulo, Brazil. ${ }^{4}$ Department of Genetics, Federal University of Pernambuco, Recife, Brazil. ${ }^{5}$ Department of Medical Images, Hematology, and Clinical Oncology, University of Sao Paulo at Ribeirao Preto Medical School, Ribeirao Preto, Sao Paulo, Brazil. ${ }^{6}$ Department of Virology, Aggeu Magalhaes Institute/Oswaldo Cruz Foundation, Recife, Brazil}

\section{Author contributions}

D.A.P-M., I.W. and A.R.L-A. conceived and designed the study, performed experiments, analyzed, and interpreted data, performed the statistical analyses, and drafted the article. J.L.C-S., P.L.F-N., L.Y.A, T.M.B, C.L.S, performed experiments, collected data, and reviewed the paper. R.F.F., F.T., E.M.R., J.J.S. and A.R.L-A. reviewed the paper. E.M.R., J.S.S. and A.R.L-A. gave final approval of the submitted version.

\section{Funding}

This investigation was supported by Fundação de Amparo à Pesquisa do Estado de São Paulo (FAPESP, Grant \#2013/08135-2). D.A.P-M. received a fellowship from FAPESP (Grant \#2017/23117-1). I.W. received a fellowship from FAPESP (Grant \#2015/09228-0). J.L.C-S. received a fellowship from FAPESP (Grant \#2016/23191-4). I.W and D.A.P-M were sponsored by the Abel Tasman Talent Program (ATTP) of the Graduate School of Medical Sciences of the University of Groningen/University Medical Center Groningen (UG/UMCG), The Netherlands.

\section{Ethics statement}

Primary patient samples were studied after informed consent and protocol approval by the Ethical Committee in accordance with the Declaration of Helsinki (registry \#12920; process number \#13496/2005; CAAE: 155.0.004.00005). Mouse experiments were performed in accordance with the national and institutional guidelines, and all experiments were approved by the Institutional Animal Care and Use Committee of the University of Sao Paulo (IACUC-USP; Process number \#067/2018)
Conflict of interest

The authors declare no competing interests.

\section{Publisher's note}

Springer Nature remains neutral with regard to jurisdictional claims in published maps and institutional affiliations.

Supplementary information The online version contains supplementary material available at https://doi.org/10.1038/s41419-021-03604-z.

Received: 18 August 2020 Revised: 8 March 2021 Accepted: 9 March 2021 Published online: 06 April 2021

\section{References}

1. Emerling, B. M. et al. MLL5, a homolog of Drosophila trithorax located within a segment of chromosome band 7q22 implicated in myeloid leukemia. Oncogene 21, 4849-4854 (2002).

2. Madan, V. et al. Impaired function of primitive hematopoietic cells in mice lacking the mixed-lineage-leukemia homolog MLL5. Blood 113, 1444-1454 (2009).

3. Sebastian, S. et al. MLL5, a trithorax homolog, indirectly regulates H3K4 methylation, represses cyclin A2 expression, and promotes myogenic differentiation. Proc. Natl Acad. Sci. USA 106, 4719-4724 (2009).

4. Ruthenburg, A. J., Allis, C. D. \& Wysocka, J. Methylation of lysine 4 on histone H3: intricacy of writing and reading a single epigenetic mark. Mol. Cell 25, 15-30 (2007).

5. Ali, M. et al. Molecular basis for chromatin binding and regulation of MLL5. Proc. Natl Acad. Sci. USA 110, 11296-11301 (2013).

6. Zhang, X., Novera, W., Zhang, Y. \& Deng, L.-W. MLL5 (KMT2E): structure, function, and clinical relevance. Cell Mol. Life Sci. 74, 2333-2344 (2017).

7. Heuser, M. et al. Loss of MLL5 results in pleiotropic hematopoietic defects, reduced neutrophil immune function, and extreme sensitivity to DNA demethylation. Blood 113, 1432-1443 (2009).

8. Damm, F. et al. Prognostic importance of histone methyltransferase MLL5 expression in acute myeloid leukemia. J. Clin. Oncol. 29, 682-689 (2011).

9. Lucena-Araujo, A. R. A. R. et al. Prognostic impact of KMT2E transcript levels on outcome of patients with acute promyelocytic leukaemia treated with alltrans retinoic acid and anthracycline-based chemotherapy: an International Consortium on Acute Promyelocytic Leukaemia study. Br. J. Haematol. 166, 540-549 (2014).

10. Yun, H. et al. Impact of MLL5 expression on decitabine efficacy and DNA methylation in acute myeloid leukemia. Haematologica 99, 1456-1464 (2014)

11. Lucena-Araujo, A. R. A. R. et al. $\triangle N p 73$ overexpression promotes resistance to apoptosis but does not cooperate with PML/RARA in the induction of an APLleukemic phenotype. Oncotarget 8, 8475-8483 (2017).

12. Boehm, D. et al. SMYD2-mediated histone methylation contributes to HIV-1 latency. Cell Host Microbe 21, 569-579.e6 (2017).

13. Subramanian, A. et al. Gene set enrichment analysis: a knowledge-based approach for interpreting genome-wide expression profiles. Proc. Natl Acad. Sci. USA 102, 15545-5550 (2005).

14. Law, C. W., Chen, Y., Shi, W. \& Smyth, G. K. voom: precision weights unlock linear model analysis tools for RNA-seq read counts. Genome Biol. 15, R29 (2014).

15. Metsalu, T. \& Vilo, J. ClustVis: a web tool for visualizing clustering of multivariate data using principal component analysis and heatmap. Nucleic Acids Res. 43, W566-W570 (2015)

16. Wang, K. et al. PML/RARa targets promoter regions containing PU.1 consensus and RARE half sites in acute promyelocytic leukemia. Cancer Cell 17, 186-197 (2010).

17. Luesink, M. et al. Chemokine induction by all-trans retinoic acid and arsenic trioxide in acute promyelocytic leukemia: triggering the differentiation syndrome. Blood 114, 5512-5521 (2009).

18. Payton, J. E. et al. High throughput digital quantification of mRNA abundance in primary human acute myeloid leukemia samples. J. Clin. Invest. 119, 1714-1726 (2009). 
19. Martens, J. H. A. et al. PML-RARa/RXR alters the epigenetic landscape in acute promyelocytic leukemia. Cancer Cell 17, 173-185 (2010).

20. Horna, P., Zhang, L., Sotomayor, E. M., Lancet, J. E. \& Moscinski, L. C. Diagnostic immunophenotype of acute promyelocytic leukemia before and early during therapy with all-trans retinoic acid. Am. J. Clin. Pathol. 142, 546-552 (2014).

21. Cancer Genome Atlas Research Network, LeyT. J., Miller, C., Raphael, DingL., \& Mungall, A. J. Genomic and epigenomic landscapes of adult de novo acute myeloid leukemia. N. Engl. J. Med. 368, 2059-2074 (2013).

22. Tasdogan, A. et al. DNA damage-induced HSPC malfunction depends on ROS accumulation downstream of IFN-1 signaling and bid mobilization. Cell Stem Cell 19, 752-767 (2016).

23. de Thé, H., Le Bras, M. \& Lallemand-Breitenbach, $\vee$. The cell biology of disease: acute promyelocytic leukemia, arsenic, and PML bodies. J. Cell Biol. 198, 11-21 (2012).

24. Arteaga, M. F., Mikesch, J.-H., Fung, T.-K. \& So, C. W. E. Epigenetics in acute promyelocytic leukaemia pathogenesis and treatment response: a TRAnsition to targeted therapies. Br. J. Cancer 112, 413-418 (2015).

25. Singh, A. A. et al. Multi-omics profiling reveals a distinctive epigenome signature for high-risk acute promyelocytic leukemia. Oncotarget 9, 25647-25660 (2018).

26. Lanotte, $M$. et al. NB4, a maturation inducible cell line with $t(15 ; 17)$ marker isolated from a human acute promyelocytic leukemia (M3). Blood. 77, 1080-1086 (1991).

27. Huynh, T. T. et al. Retinoic acid and arsenic trioxide induce lasting differentiation and demethylation of target genes in APL cells. Sci. Rep. 9, 9414 (2019).
28. Deng, L.-W., Chiu, I. \& Strominger, J. L. MLL 5 protein forms intranuclear foci, and overexpression inhibits cell cycle progression. Proc. Natl Acad. Sci. USA 101, 757-762 (2004).

29. Cheng, F. et al. RNA interference against mixed lineage leukemia 5 resulted in cell cycle arrest. Int. J. Biochem. Cell Biol. 40, 2472-2481 (2008).

30. Lucena-Araujo, A. R. et al. Combining gene mutation with gene expression analysis improves outcome prediction in acute promyelocytic leukemia. Blood 134, 951-959 (2019).

31. Klco, J. M. et al. Functional heterogeneity of genetically defined subclones in acute myeloid leukemia. Cancer Cell 25, 379-392 (2014).

32. Florean, C., Schnekenburger, M., Grandjenette, C., Dicato, M. \& Diederich, M Epigenomics of leukemia: from mechanisms to therapeutic applications. Epigenomics 3, 581-609 (2011).

33. Schenk, T. et al. Inhibition of the LSD1 (KDM1A) demethylase reactivates the all-trans-retinoic acid differentiation pathway in acute myeloid leukemia. Nat. Med. 18, 605-611 (2012).

34. Platzbecker, U. et al. Improved outcomes with retinoic acid and arsenic trioxide compared with retinoic acid and chemotherapy in non-high-risk acute promyelocytic leukemia: final results of the randomized Italian-German APL0406 trial. J. Clin. Oncol. 35, 605-612 (2017).

35. Lo-Coco, F. et al. Retinoic acid and arsenic trioxide for acute promyelocytic leukemia. N. Engl. J. Med. 369, 111-121 (2013).

36. Abaza, Y. et al. Long-term outcome of acute promyelocytic leukemia treated with all-trans-retinoic acid, arsenic trioxide, and gemtuzumab. Blood 129, 1275-1283 (2017) 